\section{Lamellolamellar sclerokeratoplasty. Where do we stand today?}

\begin{abstract}
Purpose To determine the efficacy of lamellar sclerokeratoplasty for patients with extensive corneal/corneoscleral lesions.

Methods One hundred and sixty-six eyes with extensive corneal/corneoscleral lesions of

sclerokeratoplasty at the Dr Rajendra Prasad

Centre and were followed up for at least 6 months were analysed. Patients operated on for segmental lamellar sclerokeratoplasty were not included. All the surgeries in this study were performed by a single surgeon (A.P.).
\end{abstract} different aetiology who were poor candidates for conventional lamellar keratoplasty/ penetrating keratoplasty were selected for lamellar sclerokeratoplasty.

Results Corneal clarity of $3+$ or more was achieved in 62 eyes by a single procedure. In the remaining 104 eyes graft clarity was $<2+$ due to post-surgical complications $(n=56)$ or remaining recipient deep opacity $(n=48)$.

Conclusions Astigmatism, the bugbear of final visual achievement despite a clear graft, was not a frequent problem following lamellar sclerokeratoplasty. Similarly, a gain in visual acuity to $6 / 18$ or more in $30 \%$ of eyes, cosmetic improvement in the majority of cases and prevention of recurrence of the original disease process in susceptible cases all point towards the safety and effectiveness of the procedure. Thus, lamellar sclerokeratoplasty should be considered as a management option for patients with extensive corneal/ corneoscleral lesions.

Key words Sclerokeratoplasty, Dessmarre's corneal dissector, King's clamp,

Glycerin-preserved, Mooren's ulcer,

Squamous cell carcinoma

Lamellar sclerokeratoplasty, as its name suggests, is the removal and replacement of a partial thickness of both sclera and cornea. In this study we removed a partial thickness of total cornea along with a partial thickness $(1 \mathrm{~mm})$ of sclera, and replaced the same with identical donor tissue.

\section{Patients and methods}

Between January 1977 and December 1996, a total of 166 eyes with extensive corneoscleral lesions that underwent central lamellar

\section{Surgical procedure}

The corneal lesions were outlined with a $12 \mathrm{~mm}$ trephine blade using the corneal centre as reference. A perpendicular cut on the sclera was made by pressing the trephine blade. The desired depth of the vertical cut was determined by the depth of the corneal pathology. The upper lip of the tissue to be excised was grasped firmly and a pocket was made with the help of a Paufique knife. With a Dessmarre's corneal dissector the sclera was dissected by the open technique up to the limbus all round. The lamellar limbal dissection was made carefully by the same technique. After the corneal limit had been reached the dissection was continued by the closed technique with gentle side-to-side movement. While dissecting the dissector was directed parallel to the bed of posterior stromal lamellae and no pressure was exerted directly posteriorly, to prevent penetration into the anterior chamber. If the dissected corneal bed was not sufficiently clean, the dissection was repeated at a deeper level until a clean stromal bed was attained. However, in patients who had pre-existing corneal opacity of more than $90 \%$ of corneal thickness the dissection was limited to $90 \%$ thickness irrespective of whether the opacity was central or peripheral.

To obtain a partial-thickness donor corneoscleral button, the corneoscleral tissue was fixed in a King's clamp (Fig. 1). Either fresh, M-K preserved or rehydrated glycerinpreserved donor tissue was used. The thickness varied from 0.3 to $0.4 \mathrm{~mm}$ depending on the exact thickness desired. The closed technique of dissection was followed in all cases to obtain a smooth bed. A thorough saline antibiotic wash was given to the recipient bed to remove any tissue debris.

\section{A. Panda}

Dr Rajendra Prasad Centre for Ophthalmic Sciences AllAMS New Delhi, India

Dr Anita Panda 404, Hawa Singh Block Asiad Village New Delhi 49 India

Received: 9 July 1998 Accepted in revised form: 30 November 1998 


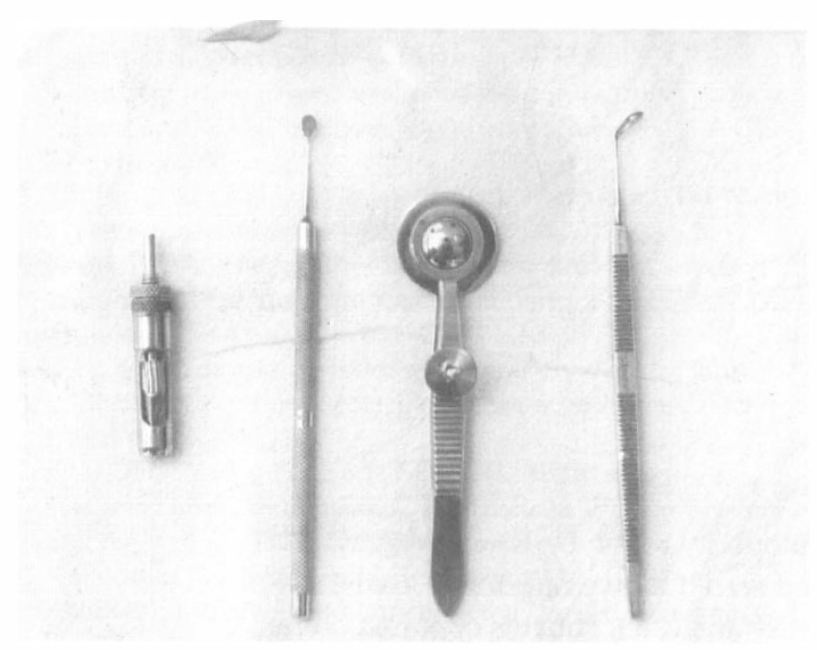

Fig. 1. Instruments for lamellar sclerokeratoplasty. From left to right: trephine, Gills knife, King's clamp, Dessmarre's corneal dissector.

The graft was fixed to the recipient bed with 16 interrupted 10-0 monofilament nylon sutures with buried knots. Antibiotic drops and a firm pressure bandage were applied at the end of surgery.

Post-operative therapy included systemic corticosteroid $1.5 \mathrm{mg} / \mathrm{kg}$ body weight once daily, together with prednisolone acetate drop 6-8 times a day. The dose of systemic corticosteroid was tapered and stopped at the end of 6 weeks, while the topical drops were continued with a frequency of two or three times daily for 6 months. Topical antibiotic was given 4 times a day for 2 weeks to prevent secondary infection. Suture removal was carried out after 6 weeks, or earlier if indicated (e.g. loose/tight suture or initiation of vascularisation). Keratometry was carried out in all patients using a Bausch \& Lomb keratometer. Graft clarity was graded by slit-lamp biomicroscopy, while refraction was carried out at the end of 6 months, whenever possible.

\section{Results}

The pre-operative diagnoses of the patients were: congenital disease 34, acquired disease 17 and posttraumatic causes 115 (Table 1). The follow-up was 6 months. The graft clarity was $3+$ or more in 62 eyes (Table 1). Graft clarity of $2+$ or less in 104 eyes was due either to remaining opacities where depth of involvement was more than $90 \%$, to post-surgical complications such as graft infection or graft vascularisation. Of 86 eyes with post-operative vascularisation, less than $90^{\circ}$ was observed in 26 eyes, $90^{\circ}-180^{\circ}$ in 44 eyes and more than $180^{\circ}$ involvement in the remaining 16 eyes (Table 2). Astigmatism of less than $3 \mathrm{D}$ was achieved in 78 eyes while only 50 eyes gained vision of $6 / 18$ or more (Table 2 ). The causes of suboptimal visual acuity (less than 6/18) in 116 eyes were pre-existing recipient opacity in 61 eyes, vascularisation occluding the visual axis in 28 eyes and graft infection leading to an opaque graft in 27 eyes (Table 3). The analysis of graft clarity in relation to the type of preservation of donor tissue revealed that of the 105 grafts where the total recipient corneal opacity was removed, graft clarity was $3+$ or more in 33 of 39,16 of 23, 6 of 22 and 7 of 21 eyes for tissues preserved in moist chamber, M-K medium < $24 \mathrm{~h}, \mathrm{M}-\mathrm{K}$ medium $>96 \mathrm{~h}$ and glycerin respectively (Fig. 2). Similarly an analysis of graft clarity in relation to recipient disease disclosed the highest percentage of clear grafts in those with congenital pathology, followed by acquired diseases, mechanical injury and lastly chemical injury (Fig. 3).

\section{Discussion}

Extensive corneal/corneoscleral lesions, though not a frequent problem in western countries, are observed in a number of cases in India. Causes include mechanical/ chemical/radiation injury to the eye, complications of lid/conjunctival/corneal surgery, extensive Mooren's ulcer, extensive neoplastic lesions of the conjunctiva and

Table 1. Graft clarity after lamellar sclerokeratoplasty in relation to recipient disease

\begin{tabular}{|c|c|c|c|c|}
\hline \multirow[b]{2}{*}{ Type of lesions } & \multirow[b]{2}{*}{ No. of eyes } & \multicolumn{3}{|c|}{ Graft clarity } \\
\hline & & $1+$ or less & $2+$ & $3+$ or more \\
\hline \multicolumn{5}{|l|}{ Congenital $(n=34)$} \\
\hline Limbal dermoid & 27 & 13 & 8 & 6 \\
\hline Corneal dermoid & 7 & 3 & 2 & 2 \\
\hline \multicolumn{5}{|l|}{ Acquired disease $(n=17)$} \\
\hline Recurrent pterygium & 3 & - & - & 3 \\
\hline Mooren's ulcer & 8 & 4 & 3 & 1 \\
\hline Extensive band-shaped keratopathy with spheroidal degeneration & 2 & - & - & 2 \\
\hline Keratoconus & 1 & - & - & 1 \\
\hline Squamous cell carcinoma & 3 & - & - & 3 \\
\hline \multicolumn{5}{|l|}{ Traumatic $(n=115)$} \\
\hline Chemical burn & 40 & 13 & 16 & 11 \\
\hline \multicolumn{5}{|l|}{ Symblepharon } \\
\hline Post-traumatic & 35 & 8 & 11 & 16 \\
\hline Post-surgical & 38 & 7 & 16 & 15 \\
\hline \multirow[t]{2}{*}{ Multiple corneal foreign bodies } & 2 & & & 2 \\
\hline & 166 & 48 & 56 & 62 \\
\hline
\end{tabular}


Table 2. Post-operative status at 6 months

\begin{tabular}{lr}
\hline Vascularisation & 80 \\
Nil & 26 \\
$<90^{\circ}$ & 44 \\
$90^{\circ}-180^{\circ}$ & 16 \\
$>180^{\circ}$ & \\
Astigmatism & 78 \\
$<3 \mathrm{D}$ & 42 \\
$3-5 \mathrm{D}$ & 6 \\
$>5$ D & 40 \\
Not measurable & \\
Visual acuity & \\
$<6 / 60$ & 35 \\
$6 / 60-6 / 24$ & 81 \\
$6 / 18$ or more & 50 \\
\hline
\end{tabular}

cornea $^{1-3}$ and finally cases of large congenital corneal/ limbal dermoid. ${ }^{4}$ These demand a large corneoscleral graft. $^{1-4}$ It is not possible to deal with these lesions by means of a conventional penetrating or lamellar keratoplasty. Moreover, an eccentric lamellar keratoplasty could not be performed due to odd surgical suture lines at the optical centre. Use of the excimer laser, though an alternative for treating such an extensive scar, may not be feasible. ${ }^{5}$ Thus management is a demanding endeavour both because of the technical difficulties and the challenging problems that usually follow a large graft. It is imperative, therefore, to define a clear-cut procedure to aid clinicians. The main goals of a largediameter lamellar sclerokeratoplasty are to remove the entire pathology, to obtain a good optical result, to avoid recurrence whenever expected and to provide mechanical support.

In the West, the incidence of lamellar corneal grafting has been reduced dramatically due to the availability of better donor tissue and finer surgical instruments.

However, in India the situation is quite different. Firstly, there is a paucity of good donor corneal tissue, and secondly the indications for keratoplasty are not the same as in western countries. Therefore, the scope of lamellar keratoplasty in India is enormous. Given the indications for the surgery a new emerging classification for lamellar keratoplasty (LK) is being followed at our centre (Table 4). Though conventional inlay LK is
Table 3. Causes of suboptimal vision $<6 / 18(\mathrm{n}=116)$

\begin{tabular}{ll}
\hline Pre-existing recipient opacity & $61(53 \%)$ \\
Vascularisation occluding the visual axis & $28(24 \%)$ \\
Graft infection leading to partially opaque graft & $27(23 \%)$ \\
\hline
\end{tabular}

classically meant for the central subtotal LK, a total LK and circular lamellar sclerokeratoplasty can be fitted into this category. Further, a large-diameter lamellar sclerokeratoplasty is also considered a form of stem cell transplantation, as it replaces not only the diseased cornea but also the diseased stem cells by healthy donor corneal tissue. However, as the total limbus with adjacent sclera is involved there is more chance of immunological reaction as compared with a small-diameter lamellar keratoplasty. Therefore, it is essential to keep these patients on prolonged post-operative corticosteroid therapy.

There is no uniformity of opinion about the preferred technique for extensive lesions. ${ }^{2,3}$ We believe that lamellar sclerokeratoplasty could be useful in such eyes due to a number of factors. Following a disease process or extensive surgical or non-surgical trauma there is every possibility of damage to the corneal surface. This denuded surface is often resurfaced by the neighbouring conjunctival epithelium. If unnoticed, the process leads to corneal neovascularisation and scarring. For normal functioning, the diseased/damaged tissue demands replacement. In a case of repeated recurrent pterygium where most procedures have failed and there is visual impairment due to pupillary occlusion, lamellar sclerokeratoplasty is a great help not only as regards visual gain but also for maintaining structural integrity and restricting the chances of recurrence. ${ }^{6}$ Extensive invasive squamous cell carcinoma of the conjunctiva and cornea that has been excised a number of times not only threatens the vision but may also further invade the deeper structures. Therefore, lamellar sclerokeratoplasty is the procedure of choice in such eyes, both to maintain structural and functional integrity and to prevent further recurrence. However, excision of the neoplastic tissue in such eyes should be always frozen-section guided. ${ }^{1,2}$

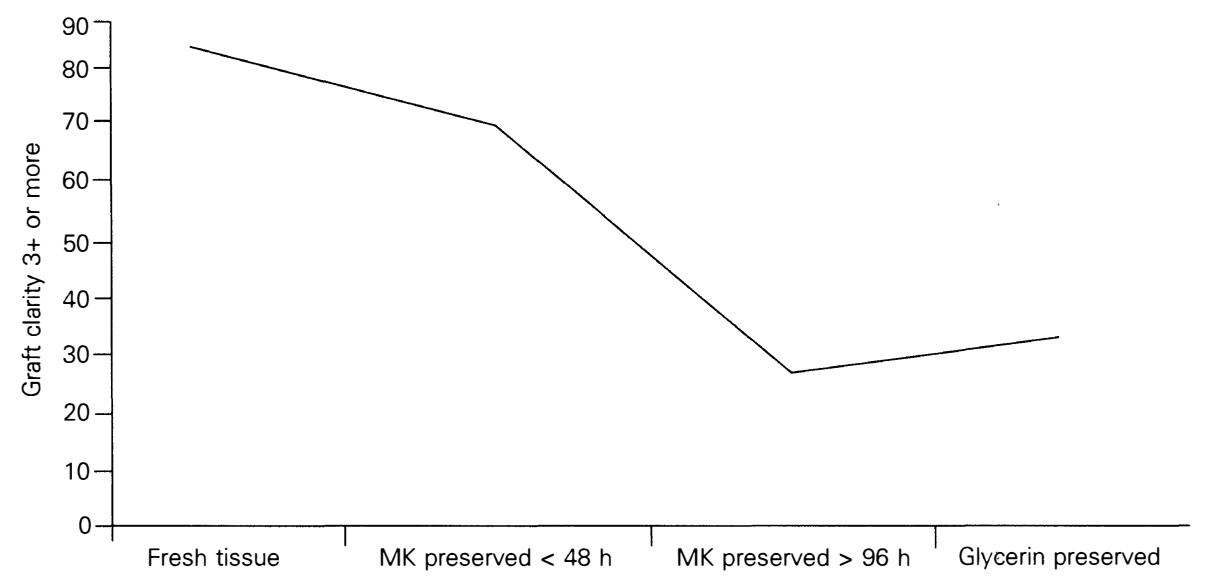

Fig. 2. Graft clarity in relation to the type of donor tissue used. 


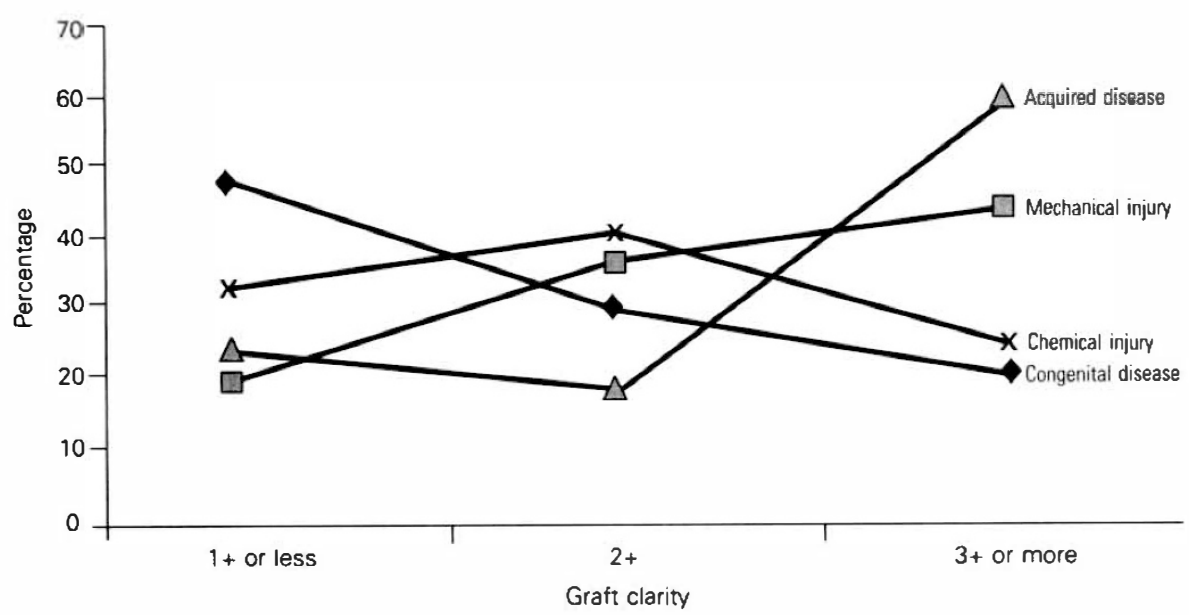

Fig. 3. Graft clarity in relation to recipient graft pathology.

All cases of trauma and Mooren's ulcer require lamellar sclerokeratoplasty for replenishment of stem cells for renewal and maintenance of structural integrity. It is also evident from our analysis of 166 eyes that maintenance of graft clarity in cases of chemical injury is poorer than in cases of diseased cornea ( $27 \%$ vs $60 \%$ ). This may indicate extensive damage to the limbal stem cells during the process of chemical injury. Moreover, it is also seen that a stable graft is more often obtained following mechanical injury than chemical injury ( $44 \%$ vs $27 \%$ ). Further, the maintenance of graft clarity in cases of acquired corneal disease is better than that following mechanical or chemical injury, indicating the presence of healthy surrounding corneal tissue beyond the lesion and also normal stem cells. However, no concrete opinion can be given regarding congenital lesions as most of these lesions are deep in nature, forcing the clinician to leave the recipient opacity and thus making graft clarity suboptimal. Sugita et al. ${ }^{7}$ in 1997 reported the removal of a full-thickness corneal lesion by deep LK. However, their study does not define the horizontal diameter of the lesion to be removed and replaced. ${ }^{7}$

Table 4. Classification of lamellar keratoplasty

A. Inlay $L K$

I. Conventional: lesions involving central/total cornea

(a) Subtotal

(b) Total

(c) Circular lamellolamellar sclerokeratoplasy (large-diameter LK)

II. Segmental: lesions not involving the pupillary area
(a) Lamellolamellar keratoplasty
(b) Lamellolamellar sclerokeratoplasty
(c) Stem cell transplant

III. Keyhole: lesions involving both peripheral/pupillary area

(a) Lamellolamellar keratoplasty

(b) Lamellolamellar sclerokeratoplasty

IV. Annular: lesions involving only the peripheral cornea

(a) Annular LK

(b) Annular stem cell transplant

B. Onlay $L K$

(a) Epikeratoplasty
Schermer et al. ${ }^{8}$ in 1986 noted that stem cells are present at the limbus and responsible for epithelial replacement and maturation. Ebato et al. ${ }^{9}$ in 1987 provided evidence that limbal epithelial cells grow much better than the peripheral and central corneal epithelia. This may be why large-diameter lamellolamellar sclerokeratoplasties survive. More recently Kuckelkorn et al. ${ }^{10}$ and Redbrake et al. ${ }^{11}$ also had encouraging results using 11-12 mm diameter penetrating keratoplasty for alkali-burned corneas. However, $33 \%$ of the eyes from their series showed rejection at between 2 and 3 months post-operatively.

Using the procedure in a number of cases we observed that a $12 \mathrm{~mm}$ diameter lamellar sclerokeratoplasty is feasible for extensive corneoscleral lesions of any aetiology. Results cannot be compared with cases of congenital limbal/corneal dermoid as the suboptimal graft clarity in these patients is due to posterior corneal opacity in addition to limbal stem cell damage. Achievement of graft clarity of $3+$ or more in about $37 \%$ of cases, visual acuity of $6 / 18$ or more in $30 \%$, and above all astigmatism of less than $3 \mathrm{D}$ in $64 \%$ of eyes all indicate the safety of the procedure. In comparison a congenital lesion such as a limbal or corneal dermoid provided a clear graft in $50 \%$ of eyes; the visual acuity was not so encouraging, apparently due to associated amblyopia.

The desired outcome after lamellar sclerokeratoplasty was certainly dependent on the nature of the recipient's disease. Interestingly, given the importance of chemical injury and congenital problems of the cornea as major indications for lamellar sclerokeratoplasty, physical success (clarity) was markedly less than that in cases of acquired diseases and mechanical injuries.

Though none of our cases have reported to us at the acute stage of graft rejection, peripheral vascularisation of mild to moderate grade was experienced in $38 \%$ of eyes and severe vascularisation was seen in another $10 \%$ of eyes. Thus the presence of vascularisation and suboptimal graft over a large number of cases could not rule out the rejection episode. 
It is also noted that fresh donor cornea and cornea freshly preserved in $\mathrm{M}-\mathrm{K}$ medium maintain better graft clarity than 4-day-old corneas preserved in $\mathrm{M}-\mathrm{K}$ medium and glycerin-preserved corneas. Better results obtained using glycerin-preserved corneas may indicate a loss of antigenicity; however, this is difficult to prove. Further, it is unclear whether the existing stem cells lost their property of repopulation when preserved for a long time in $\mathrm{M}-\mathrm{K}$ medium.

In conclusion, lamellar sclerokeratoplasty is recommended for treating patients who have extensive corneal or eccentric corneoscleral lesions that are not amenable to either conventional lamellar or penetrating keratoplasty. Further, to obtain healthy stem cells, fresh or freshly preserved corneoscleral tissue is preferred over long-term glycerin-preserved corneas or intermediateterm M-K-preserved cornea. Even though graft clarity and visual acuity are suboptimal in a large number of eyes the procedure does not preclude subsequent conventional penetrating keratoplasty.

\section{References}

1. Panda A, Sharma N. Frozen section guided excision of squamous cell carcinoma of conjunctiva and cornea ophthalmic surgery. News Int 1996;7:42.
2. Panda A, Sharma N, Ghose S, Sen S. FSGE and lamellar sclerokeratoplasty for recurrent squamous cell carcinoma of conjunctiva and cornea. In: Advances in cornea research. New York: Plenum Press, 1996:143-9.

3. Arora R, Panda A, Kumar H. Lamellar sclerokeratoplasty for xeroderma pigmentosa. J Pediatr Ophthalmol Strabismus 1990;27:80-3.

4. Mohan M, Panda A. Lamellar keratoplasty for limbal dermoid. In J Ophthalmol 1981;29:137-40.

5. Massimo B, Halliday BL, Robert CA, et al. Precarved lyophilised tissue for lamellar keratoplasty in recurrent pterygium. Am J Ophthalmol 1986;102:222-7.

6. Cann DB, Downie NA, Young IM, et al. Excimer laser lamellar keratoplasty. Ophthalmology 1995;23:189-94.

7. Sugita J, Kondo I. DLK with complete removal of pathological stroma for vision improvement. Ophthalmology 1997;81:184-8.

8. Schermer A, Galvin S, Sun TT. Differentiation expression of a major 64K corneal keratin. J Cell Biol 1986;103:49-62.

9. Ebato B, Friend J, Thoft RA. Comparison of peripheral and central human corneal epithelium in tissue culture. Invest Ophthalmol Vis Sci 1987;28:1430-6.

10. Kuckelkorn R, Redbrake C, Schrage NF, Reim M. Keratoplasty with 11-12 mm diameter for management of severely chemically burned eyes. Ophthalmology 1993;90:683-7.

11. Redbrake C, Buchal V, Reim M. Keratoplasty with a scleral rim after alkali burn. Klin Monatsbl Augenheilkd 1996;208:145-51. 